Pis'ma v ZhETF

\title{
Phase Transition in Strongly Degenerate Hydrogen Plasma
}

\author{
V.S. Filinov*, V.E.Fortov*, M. Bonitz ${ }^{* * 1)}$, and P.R. Levashov* \\ *Institute for High Energy Densities RAS, 127412 Moscow, Russia \\ ** Fachbereich Physik, Universität Rostock, D-18051 Rostock, Germany
}

Submitted 20 August 2001

\begin{abstract}
Direct fermionic path-integral Monte-Carlo simulations of strongly coupled hydrogen are presented. Our results show evidence for the hypothetical plasma phase transition. Its most remarkable manifestation is the appearance of metallic droplets which are predicted to be crucial for the electrical conductivity allowing to explain the rapid increase observed in recent shock compression measurments.
\end{abstract}

PACS: 52.25.Kn; 52.65.Pp

Hydrogen at high pressures remains the subject of many investigations, see e.g. [1, 2] for an overview. 'Many interesting phenomena, such as a metal-insulator transition (MIT), Mott effect and a plasma phase tran'sition (PPT) have been predicted. They occur in situations where both, quantum and Coulomb effects are important, making a theoretical analysis difficult. Among 'the most promising theoretical approaches to such systems is the path-integral quantum Monte Carlo (PIMC) 'method [3, 4] which has seen remarkable recent progress, e.g. [4, 5. However, for Fermi systems, these simulations are substantially hampered by the so-called fermion sign problem. Additional assumptions such as 'fixed node and restricted path concepts have been intro'duced to overcome this difficulty [四. It can be shown, however, that such assumptions do not reproduce the 'correct ideal Fermi gas limit [6].

Recently, we have presented a new path integral 'representation which avoids additional approximations (direct path integral Monte-Carlo, DPIMC) which has 'successfully been applied to strongly coupled hydrogen [7, 8, 9, see below. In this work we apply the DPIMC method to the analysis of dense liquid hydro'gen in the region of the hypothetic plasma phase transition [10, 1, 11, 12]. Computing the equation of state and the internal energy, we find clear indications for the existence of the PPT - its first confirmation by a firstprinciple method. It is shown that the PPT manifests itself by the formation of large metallic droplets which are crucial for the plasma transport properties.

It is well known that the thermodynamic properties of a quantum system are fully determined by the partition function $Z$. For a binary mixture of $N_{e}$ electrons and $N_{i}$ protons, $Z$ can be written as

\footnotetext{
1) e-mail: michael.bonitz@physik.uni-rostock.de
}

$$
\begin{aligned}
Z\left(N_{e}, N_{i}, V, \beta\right) & =Q\left(N_{e}, N_{i}, \beta\right) / N_{e} ! N_{i} ! \\
Q\left(N_{e}, N_{i}, \beta\right) & =\sum_{\sigma} \int_{V} d q d r \rho(q, r, \sigma ; \beta) .
\end{aligned}
$$

Here, $q \equiv\left\{\mathbf{q}_{1}, \mathbf{q}_{2}, \ldots, \mathbf{q}_{N_{i}}\right\}$ are the coordinates of the protons and and $\sigma=\left\{\sigma_{1}, \ldots, \sigma_{N_{e}}\right\}$ and $r \equiv$ $\left\{\mathbf{r}_{1}, \ldots, \mathbf{r}_{N_{e}}\right\}$ are the electron spins and coordinates, respectively, and $\beta=1 / k_{B} T$. The density matrix $\rho$ in Eq. (11) is represented in the common way by a path integral [13]:

$\rho(q, r, \sigma ; \beta)=\frac{1}{\lambda_{i}^{3 N_{i}} \lambda_{\Delta}^{3 N_{e}}} \sum_{P}( \pm 1)^{\kappa_{P}} \int_{V} d r^{(1)} \ldots d r^{(n)} \times(2)$
$\rho\left(q, r, r^{(1)} ; \Delta \beta\right) \ldots \rho\left(q, r^{(n)}, \hat{P} r^{(n+1)} ; \Delta \beta\right) \mathcal{S}\left(\sigma, \hat{P} \sigma^{\prime}\right)$,

where $\Delta \beta \equiv \beta /(n+1)$ and $\lambda_{\Delta}^{2}=2 \pi \hbar^{2} \Delta \beta / m_{e}$. Further, $r^{n+1} \equiv r^{n}, \sigma^{\prime}=\sigma$; i. e., the electrons are represented by fermionic loops with the coordinates (beads) $[r] \equiv\left[r, r^{(1)}, \ldots, r^{(n)}, r\right]$. The electron spin gives rise to the spin part of the density matrix $\mathcal{S}$, whereas exchange effects are accounted for by the permutation operator $\hat{P}$ and the sum over the permutations with parity $\kappa_{P}$.

Following [3], we use a modified representation (3) of the high-temperature density matrices on the r.h.s. of Eq. (2) which is suitable for efficient direct fermionic PIMC simulation of plasmas. With the error of the order $\epsilon \sim(\beta R y)^{2} \chi /(n+1)$ vanishing with a growing number of beads, we obtain the approximation

$$
\begin{aligned}
& \sum_{\sigma} \rho(q, r, \sigma ; \beta)=\frac{1}{\lambda_{i}^{3 N_{i}} \lambda_{\Delta}^{3 N_{e}}} \sum_{s=0}^{N_{e}} \rho_{s}(q,[r], \beta) \\
& \rho_{s}(q,[r], \beta)=\frac{C_{N_{e}}^{s}}{2^{N_{e}}} e^{-\beta U(q,[r], \beta)} \prod_{l=1}^{n} \prod_{p=1}^{N_{e}} \phi_{p p}^{l} \operatorname{det}\left|\psi_{a b}^{n, 1}\right|_{s}, \\
& U(q,[r], \beta)=U^{i}(q)+\sum_{l=0}^{n} \frac{U_{l}^{e}([r], \beta)+U_{l}^{e i}(q,[r], \beta)}{n+1}
\end{aligned}
$$


where $\chi$ is the degeneracy parameter and $U^{i}, U_{l}^{e}$ and $U_{l}^{e i}$ denote the sum of the binary Kelbg potentials $\Phi^{a b}$ [14, 15] between protons, electrons at vertex " $l$ ", and electrons (vertex "l") and protons, respectively.

In Eq. (3), $\phi_{p p}^{l} \equiv \exp \left[-\pi\left|\xi_{p}^{(l)}\right|^{2}\right]$ arises from the kinetic energy part of the density matrix of the electron with index $p$, and we introduced dimensionless distances between neighboring vertices on the loop, $\xi^{(1)}, \ldots \xi^{(n)}$. Finally, the exchange matrix is given by

$$
\begin{gathered}
\left\|\psi_{a b}^{n, 1}\right\|_{s} \equiv|| \exp \left\{-\frac{\pi}{\lambda_{\Delta}^{2}}\left|\left(r_{a}-r_{b}\right)+y_{a}^{n}\right|^{2}\right\} \|_{s}, \\
\text { with } y_{a}^{n}=\lambda_{\Delta} \sum_{k=1}^{n} \xi_{a}^{(k)},
\end{gathered}
$$

where the subscript $s$ denotes the number of electrons having the same spin projection. From the above expressions (11)-(3) one readily computes the internal energy and the equation of state:

$$
\begin{aligned}
& \beta E=\frac{3}{2}\left(N_{e}+N_{i}\right)-\beta \frac{\partial \ln Q}{\partial \beta}, \\
& \beta p=\frac{\partial \ln Q}{\partial V}=\left[\frac{\alpha}{3 V} \frac{\partial \ln Q}{\partial \alpha}\right]_{\alpha=1} .
\end{aligned}
$$

In our simulations we used $N_{e}=N_{i}=50$ and $n=20$. To test the MC procedure, we have considered a mixture of ideal degenerate electrons and classical protons for which the thermodynamic quantities are known analytically. The agreement, up to the degeneracy parameter $\chi$ as large as 10 , has been very good and improved with increasing number of particles [7]. Further, the method was successfully tested in applications to electrons in a harmonic trap [16]. For the case of interacting electrons and protons in dense hydrogen we have previously performed a series of calculations over a wide range of the classical coupling parameter $\Gamma$ and degeneracy $\chi$ for temperatures $T \geq 10,000 \mathrm{~K}$. The analysis of the results has clearly shown a number of interesting phenomena, such as formation and decay of hydrogen bound states [8, 9, 15], including hydrogen atoms, molecules, molecular ions, clusters and futher, at high densities, pairing of electrons and ordering of protons into a Wigner crystal [9].

In this work we present new results which concentrate on the hypothetical plasma phase transition [10. For this purpose, we analyze the plasma properties and compute the equation of state (5) and internal energy (4) of dense hydrogen along two isotherms, $T=10^{4} \mathrm{~K}$ and $5 \cdot 10^{4} \mathrm{~K}$. Fig. 1 shows pressure and energy vs. density at $T=5 \cdot 10^{4} \mathrm{~K}$. For comparison, we also include the results for an ideal plasma. As expected, due to Coulomb interaction and bound state formation, the nonideal plasma results are below the ideal ones. We mention that our results are in a good agreement with restricted path integral calculations of Militzer and Ceperley (Fig.1a contains available data points for a slightly higher temperature of $6.25 \cdot 10^{4} \mathrm{~K}$ [17. For higher temperatures the agreement is very good 15 . Most importantly, at this temperature the pressure increases monotonically with density, and at high densities a continuous increase of the degree of ionization (Mott effect) is found.

However, at $T=10^{4} \mathrm{~K}$ the properties of the hydrogen plasma change qualitatively, cf. Figs. 2,3. While the overall trend of the pressure, Fig. 2.a, is still a monotonic increase, in the density region of $0.1 \ldots 1.5 \mathrm{~g} / \mathrm{cc}$ the plasma exhibits unusual behavior. Inside this region the Monte Carlo simulations do not converge to an equilibrium state, the pressure strongly fluctuated and reached even negative values. Such a behavior is typical for Monte-Carlo simulations of metastable systems. Note that no such peculiarities appear for densities below and above this interval as well as for the isotherm $T=5 \cdot 10^{4} \mathrm{~K}$ and for higher temperatures.

These facts suggest that our simulations have encountered the plasma phase transition predicted by many chemical models of partially ionized hydrogen, e.g. 10, 1, 11, 12. According to these models, this is a firstorder transition with two coexisting phases of different degree of ionization. While canonical Monte Carlo simulations do not yield the coexisting phases and the coexistence pressure directly, they allow to analyze in detail the actual microscopic particle configurations. A typical particle arrangement inside the instability region, $T=10^{4} \mathrm{~K}$ and $\rho=0.3346 \mathrm{~g} / \mathrm{cc}$, is shown in Fig. 3. Obviously, the protons arrange themselves into large clusters (droplets) with the electrons (the piecewise linear lines show their closed fermionic path) being fairly delocalized over the cluster. This is a clear precurser of the metal-like state which is found in the simulations for densities above the instability region.

As mentioned above, the plasma phase transition appears in many chemical models in the same densitytemperature range. However, these simple approaches become questionable in the region of pressure ionization and dissociation where a consistent treatment of all possible pair interactions, including charge-charge, neutral-neutral and charge-neutral, is crucial. Furthermore, these approaches neglect larger bound aggregates such as clusters which our simulations reveal to be crucial in the metastable region. We mention that indirect indications for a phase transition have been found in recent density functional studies [20. In this work the 
thermodynamic properties of hydrogen in the metallic phase were computed (see data points in Fig. 2) and enhanced long-wavelength ion density fluctuations were observed as the density was reduced to $\rho=0.799 \mathrm{~g} / \mathrm{cc}$ (the lowest density explored). This led to unusual behavior of the ion-ion structure factor and the effective potential which the authors of Ref. [20] interpreted as a possible precursor to an incipient metal-to-insulator transition.

Our simulations suggest that the existence of the PPT should have a noticable influence on the transport properties. In fact, when the density changes from $0.1 \ldots 1.5 \mathrm{~g} / \mathrm{cc}$, hydrogen transforms from a neutral into a metallic fluid. Accordingly, the electrical conductivity should increase rapidly. Indeed, shock compression experiments have revealed a dramatic increase of the electrical conductivity by $4-5$ orders of magnitude in a very narrow density range of $0.3-0.5 \mathrm{~g} / \mathrm{cc},[18,19]$. Theoretical models so far cannot reproduce this behavior correctly, predicting either a too early (hopping conductivity in the molecular fluid) or too late (free electron conductivity) increase 21. But as the experimental data (black circles and crosses in Fig. 2.a) are located right inside the $\mathrm{PPT}$ region, one has to take into account a third conductivity mechanism - charge transport via electron hopping between individual metal-lik droplets. Obviously, this mechanism will be effective inbetween the regions where the two other effects dominate and thus should allow for a much better agreement with the experiments.

Finally, we mention that our simulations predict a PPT for pure hydrogen plasma only. In contrast, no PPT was found for a binary mixture of $25 \%$ of helium and $75 \%$ of hydrogen (cf. Fig. 2).

In summary, we presented direct path integral Monte Carlo simulations of dense fluid hydrogen in the region of the metal-insulator transition (MIT). Our results show evidence for the plasma phase transition which, to the best of our knowledge, is its first prediction by a first principle theory. Most importantly, we found clear evidence for the formation of large metallic droplets which are predicted to play a crucial role in transport and optics in the region of the MIT at low temperatures. In further investigations we will focus on a more precise analysis of the MIT and the plasma phase transition, including determination of its critical point and of the transport and optical properties of the droplets.

We are greatful to W. Ebeling, D. Kremp, and W. D. Kraeft and S. Trigger for stimulating discussions. We also wish to thank D. Ceperley and B. Militzer for useful critical remarks.
1. W. D. Kraeft, D. Kremp, W. Ebeling and G. Röpke, Quantum Statistics of Charged Particle Systems, Akademie-Verlag, Berlin, 1986.

2. Strongly Coupled Coulomb Systems, edited by G. Kalman, Pergamon Press, 1998.

3. V.M. Zamalin, G. E. Norman and V.S. Filinov, The Monte-Carlo Method in Statistical Thermodynamics, Nauka, Moscow, 1977.

4. D. Ceperley, in The Monte Carlo and Molecular Dynamics of Condensed Matter Systems, edited by K. Binder and G. Ciccotti, SIF, Bologna, 1996, pp. 447-482.

5. Classical and Quantum Dynamics of Condensed Phase Simulation, edited by B. J. Berne, G. Ciccotti, and D. F. Coker, World Scientific, Singapore, 1998.

6. V.S. Filinov, J. Phys. A 34, 1665 (2001).

7. V.S. Filinov, P. R. Levashov, V. E. Fortov, and M. Bonitz, in Progress in Nonequilibrium Green's Functions, edited by M. Bonitz, World Scientific, Singapore, 2000.

8. V.S. Filinov, V.E. Fortov, M. Bonitz, and D. Kremp, Physics Letters A 274, 228 (2000).

9. V.S. Filinov, M. Bonitz, and V. E. Fortov, JETP Letters 72, 245 (2000).

10. G.E. Norman, and A.N. Starostin, TWT 8, 413 (1970).

11. M. Schlanges, M. Bonitz, and A. Tschttschjan, Contrib. Plasma Phys. 35, 109 (1995).

12. D. Saumon, and G. Chabrier, Phys. Rev. A 46, 2084 (1992)

13. R.P. Feynman, and A.R. Hibbs, Quantum Mechanics and Path Integrals, McGraw-Hill, New York, 1965.

14. G. Kelbg, Ann. Phys. (Leipzig) 12, 219 (1963) \& 13, 354 (1963) \& 14, 394 (1964).

15. For a detailed discussion, see V.S. Filinov, M. Bonitz, W. Ebeling and V.E. Fortov, Plasma Phys. Cont. Fusion 43, 743 (2001).

16. A. Filinov, M. Bonitz, and Yu.E. Lozovik, Phys. Rev. Lett. 86, 3851 (2001).

17. B. Militzer, and D. M. Ceperley, Phys. Rev. E 63, 066404 (2001).

18. S. T. Weir, A. C. Mitchell, and W. J. Nellis, Phys. Rev. Lett. 76, 1860 (1996).

19. V. Ya. Ternovoi, A.S. Filimonov, V.E. Fortov, et al., Physica B 265, 6 (1999).

20. H. Xu, and J. P. Hansen, Phys. Rev. E 57, 211 (1998).

21. R. Redmer, G. Röpke, S. Kuhlbrodth, and H. Reinholz, Contrib. Plasma Phys. 41, 163 (2001).

Fig. 1. (a) Pressure and (b) internal energy for hydrogen plasma at $T=5 \cdot 10^{4} \mathrm{~K}$ vs. density. $1-$ direct PIMC simulation of this work, 2 -ideal plasma, 3 - restricted PIMC computations at $T=6.25 \cdot 10^{4} \mathrm{~K}$ [17. 
Fig. 2. (a) Pressure (1-5) and electrical conductivity $(6,7)$ and (b) internal energy for hydrogen at $T=10^{4} \mathrm{~K}$ vs. density. 1 - direct PIMC simulation of this work, 2 -ideal plasma, 3 - direct PIMC simulation of a mixture consisting of $25 \%$ helium and $75 \%$ hydrogen, 4 - density functional theory [20], 5 restricted PIMC computations [17], 6, 7 - electrical conductivity of hydrogen (right axis), $6-[18,7-19]$.

Fig. 3. Snapshot of a Monte-Carlo cell at $T=10^{4} \mathrm{~K}$ and $\rho=0.3346 \mathrm{~g} / \mathrm{cc}$. Black circles are protons, dark and light broken lines are representations of electrons as fermionic loops with different spin projections. 


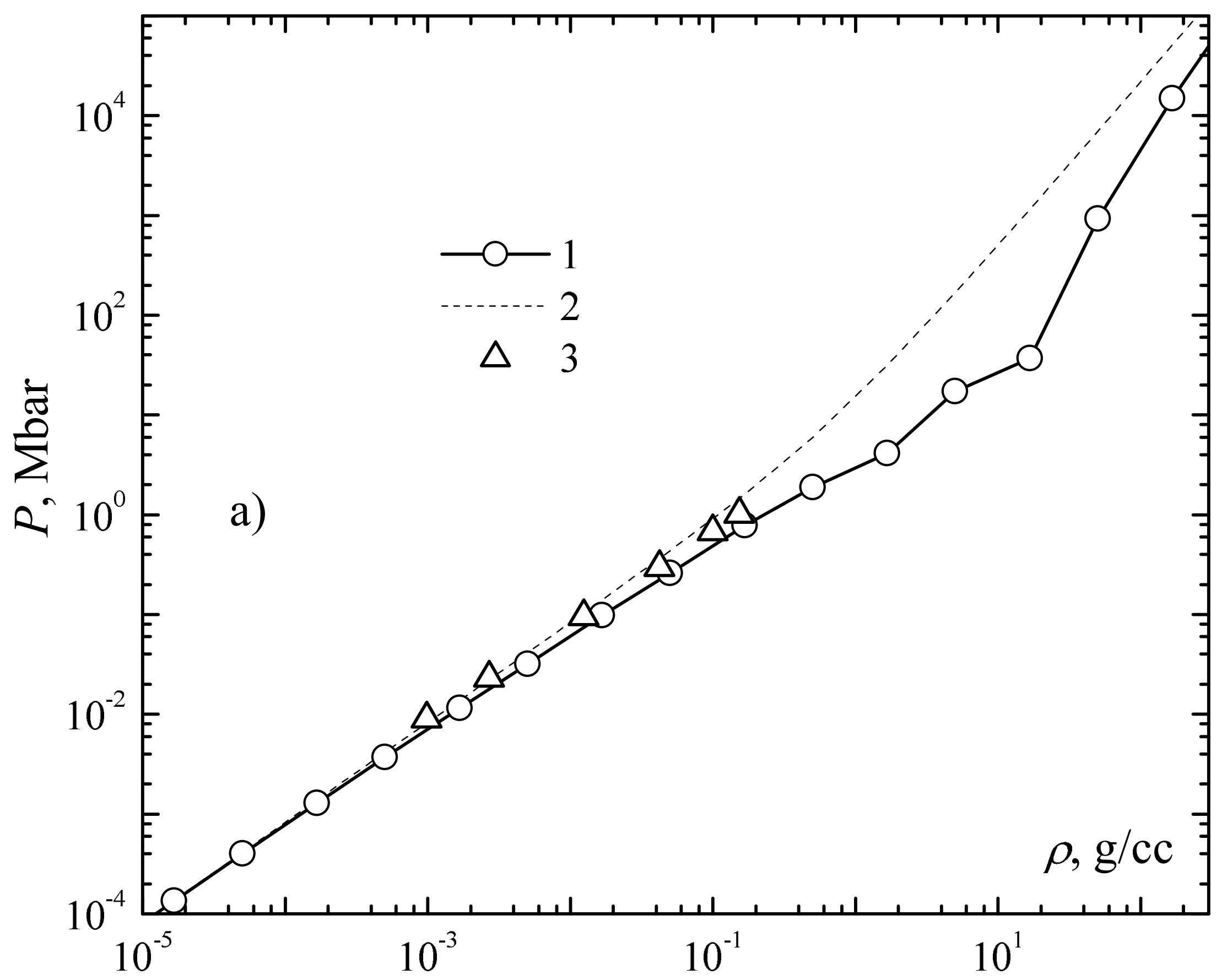




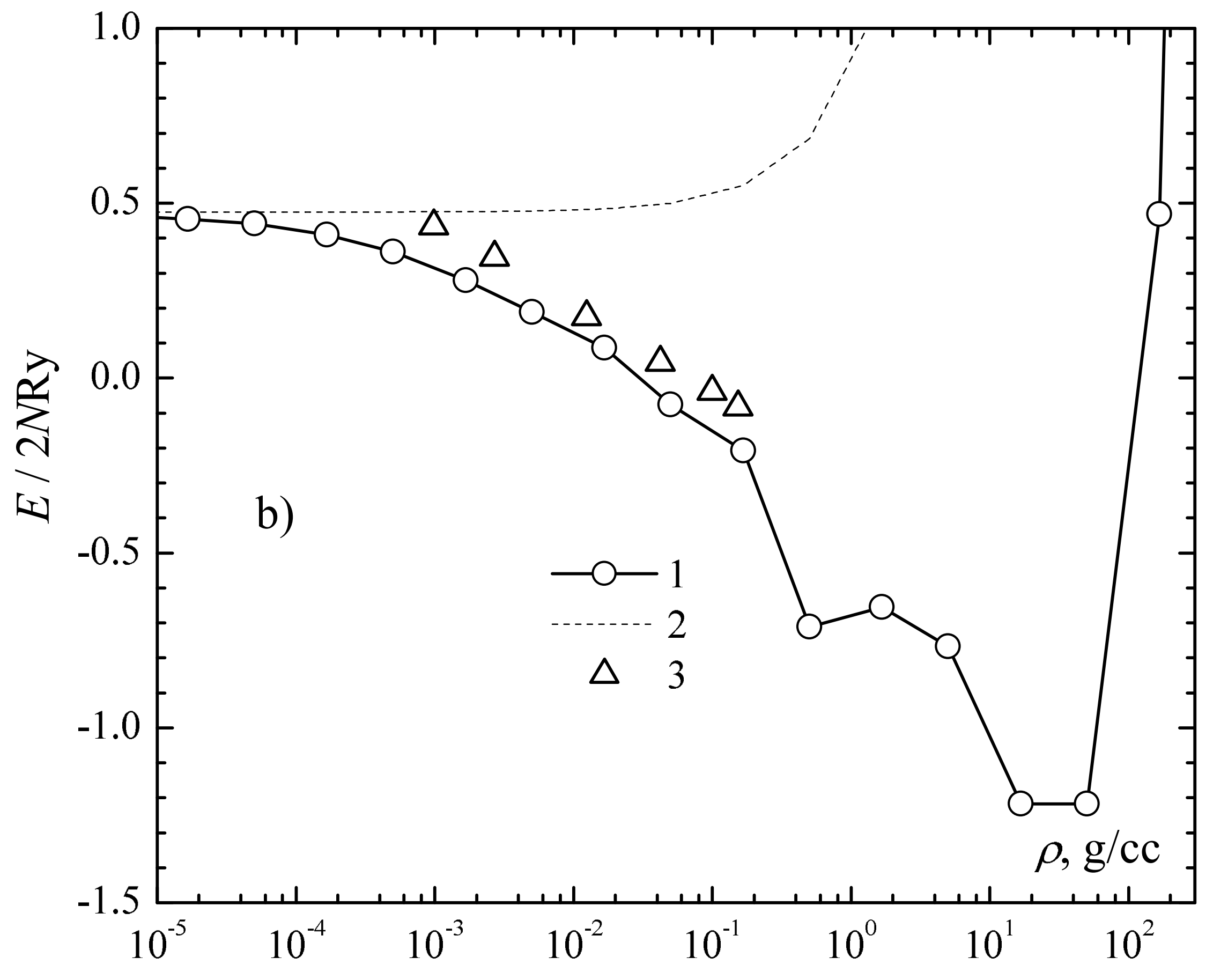




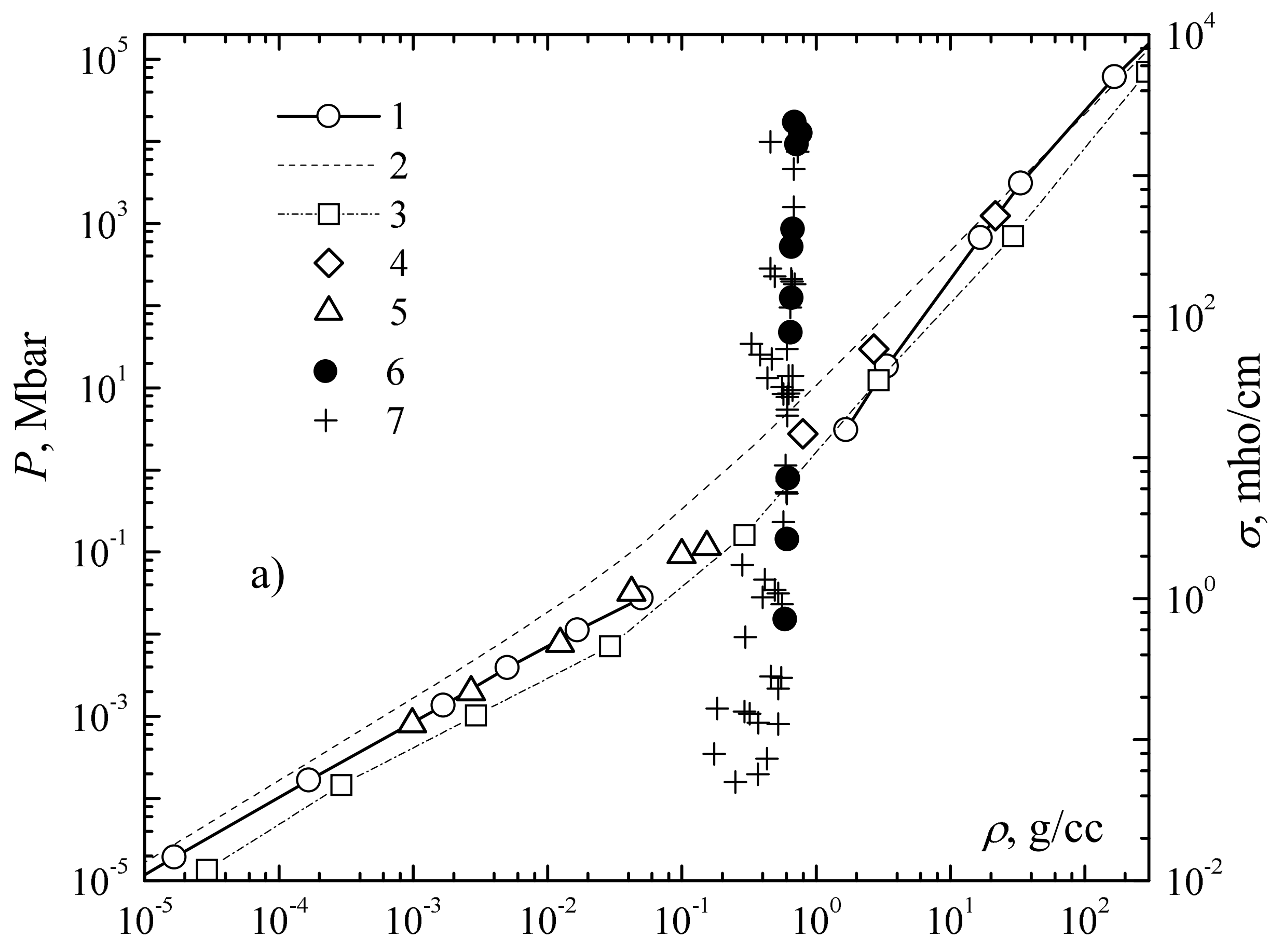




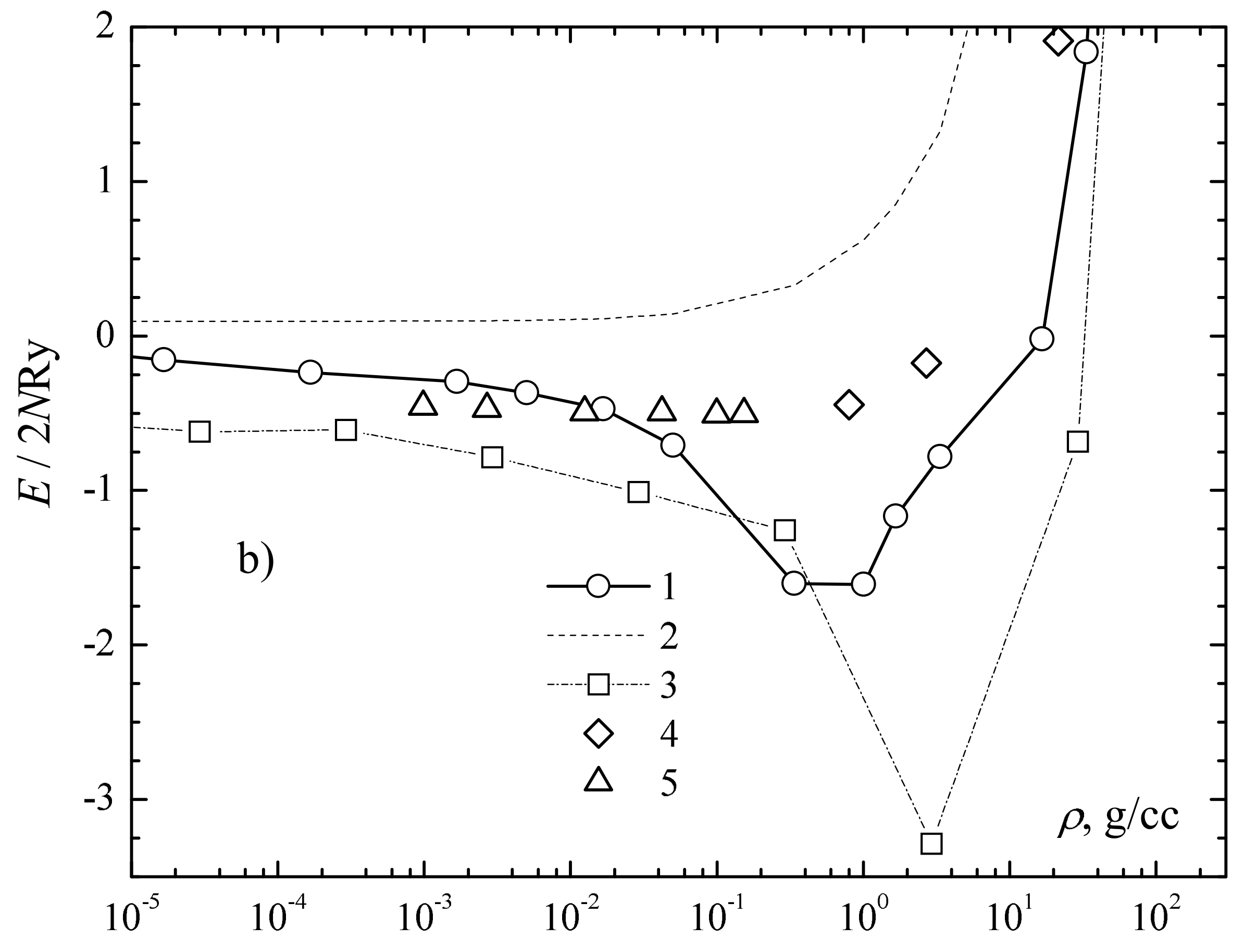




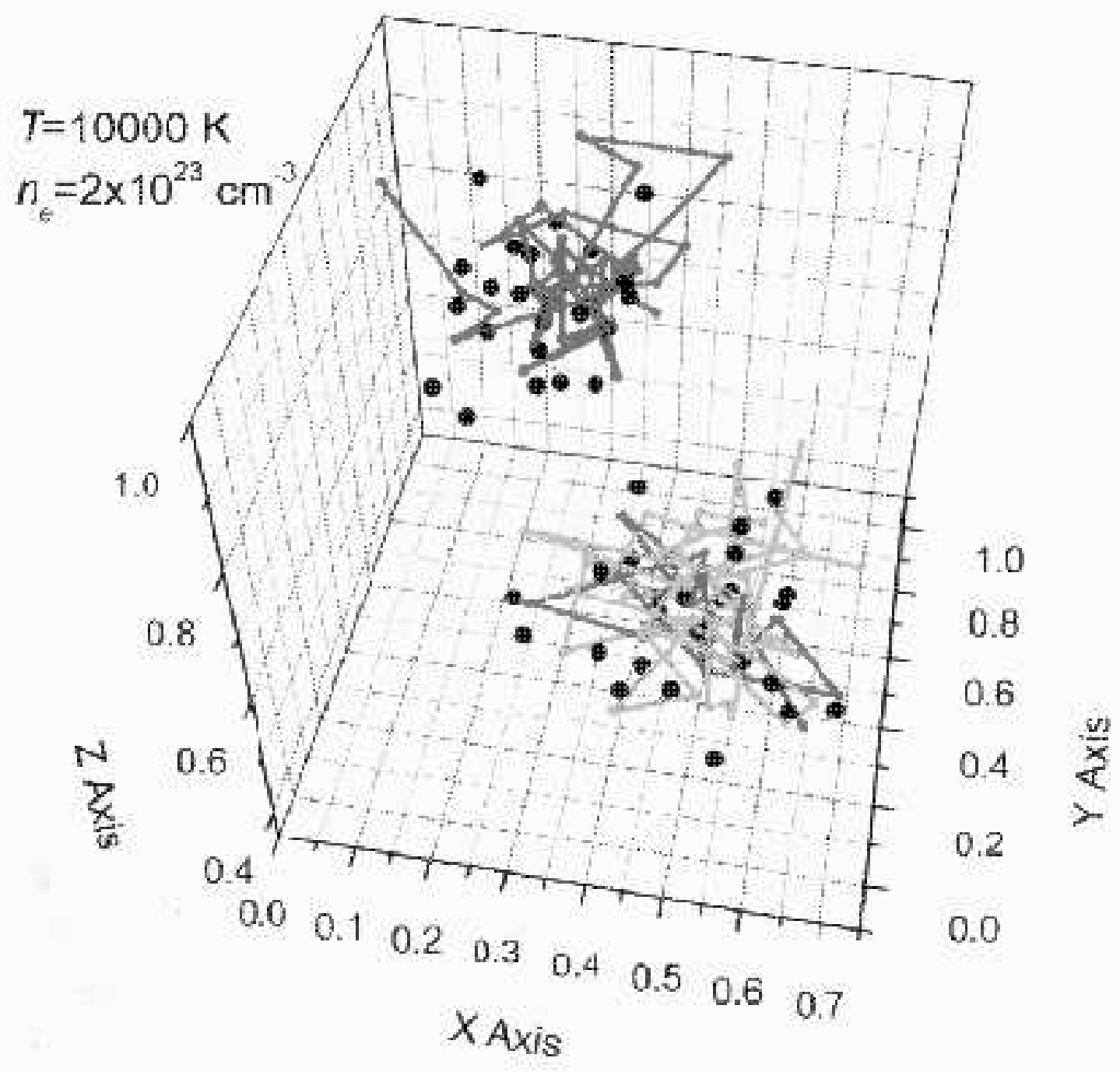

